
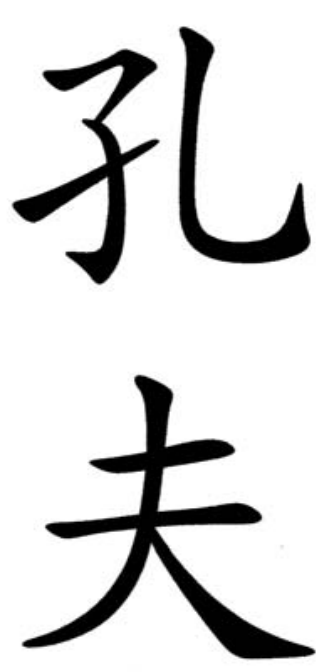

ESTATUA CONFUCIANA

en el Museo Histórico

Chino de San Diego.

Fue Regalada POR El EX

Ministro de EduCACión

KaO WeI-FAng.

Cortesía del Museo histórico

Chino de San Diego

www.sdchm.org

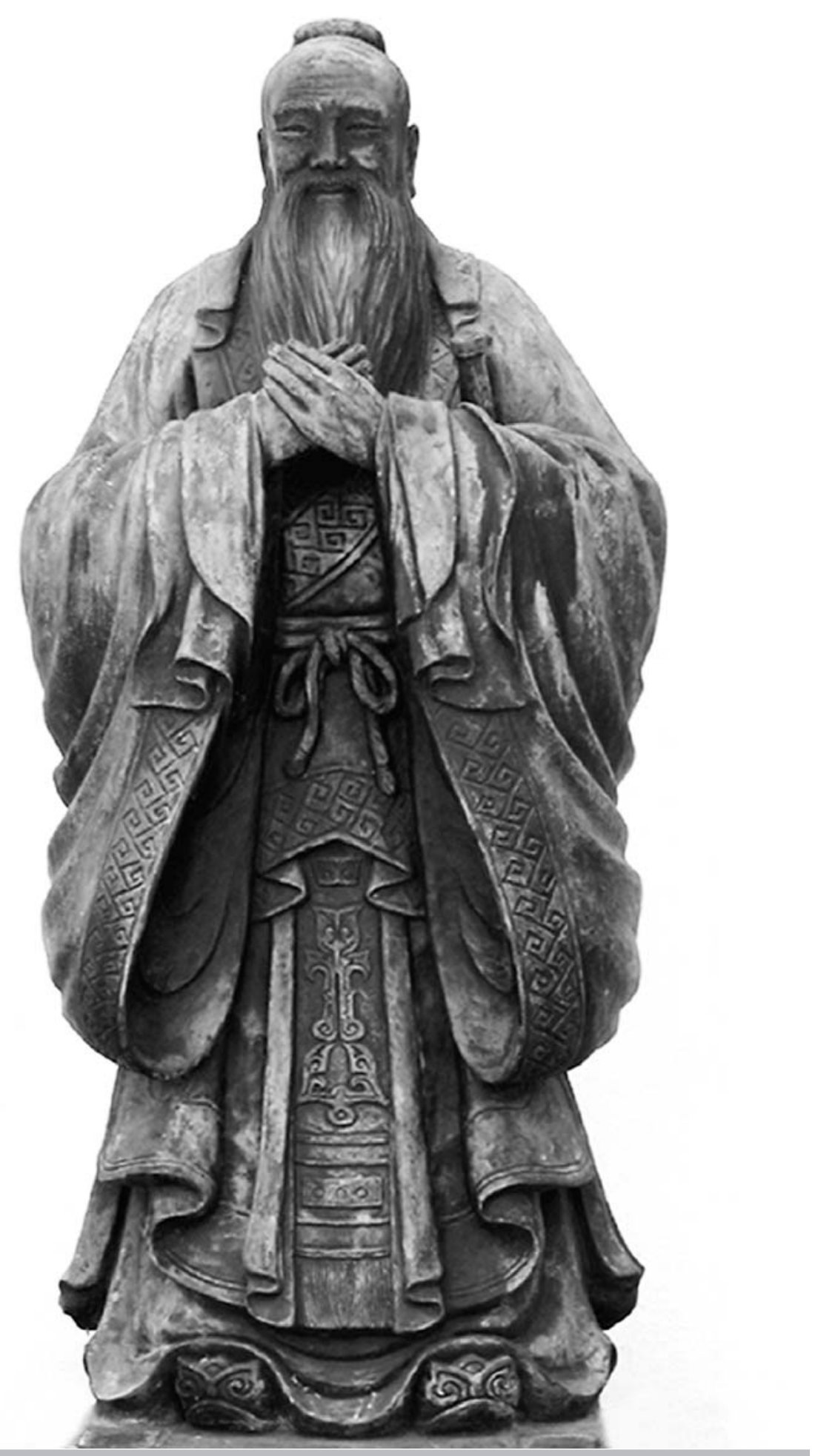

\section{Resumen}

El confucianismo ha sido un elemento base en la vida política y social de muchos países asiáticos. Su influencia se extiende por instituciones y prácticas en cada nivel de la actividad humana. Las artes marciales también se han beneficiado de esta filosofía, y el legado tradicional confuciano continúa influyendo en las prácticas modernas. Este artículo pone de relieve sucintamente algunas figuras y acontecimientos cruciales, describe conceptos relevantes y centrales de la filosofía confuciana, y por último muestra aplicaciones paradigmáticas para las artes marciales de hoy en día. Los artistas marciales modernos pueden lograr la comprensión de la visión tradicional confuciana y profundizar en el significado de las artes marciales contemporáneas. 


\title{
El Confucianismo
}

\section{Y LAS TRADICIONES}

\section{MARCIALES ASIÁTICAS}

\author{
C. Alexander Simpkins \& Annellen M. Simpkins
}

STS

El mayor aprendizaje echa raíces en la clarificación de la forma en que la inteligencia aumenta a través del proceso de mirar directamente en nuestro propio corazón y actuar de acuerdo a ello.

Pound, 1951: 27

\section{Introducción}

La filosofía confuciana ha tenido un efecto profundo en la política y sociedad asiática durante muchos siglos, pero sus efectos también se han sentido en el mundo entero. Confucio fue una grandiosa figura de la historia universal. Sus ideas inspiran a que se manifieste la mejor naturaleza de toda persona, y quizá esto es lo que hace al Confucianismo tan importante para la humanidad. Voltaire expresó el significado de esta filosofía cuando dijo: "Materializar las teorías de Confucio daría lugar al período más feliz y más valioso de la historia humana" (Chang, 1957: 110).

Uno de los legados más prolíficos del Confucianismo puede encontrarse en la práctica de las artes marciales. Así cómo China, Corea, y Japón integraron el Confucianismo en el mismo corazón de sus culturas, sus tradiciones marciales se han enriquecido por la filosofía confuciana como parte integral de la práctica.

\section{Raíces Confucianas}

Kong Qiu (551-479 a.C.), conocido como Maestro Kong (Kong Fuzi, posteriormente latinizado a Confucio), es honrado como el "Primer Maestro" de China y fundador del Confucianismo. Insistía en que no fue él quien creó de las ideas que enseñaba; en lugar de eso pensaba en sí mismo como un emisario de antiguas verdades. Confucio se ocupó del problema de la condición humana. Fijó su atención en el Dao de la Humanidad, el mundo humano de la acción en las relaciones sociales. A través de su dedicación completa a los ideales últimos de la vida y del aprendizaje, la enseñanza de Confucio transcendió la esfera de su propia vida para convertirse, para siempre, en un pilar axiomático de la gran civilización China.

Todas las fotografías, salvo indicación expresa, son cortesía de C.A. Simpkins y A.M. Simpkins. 


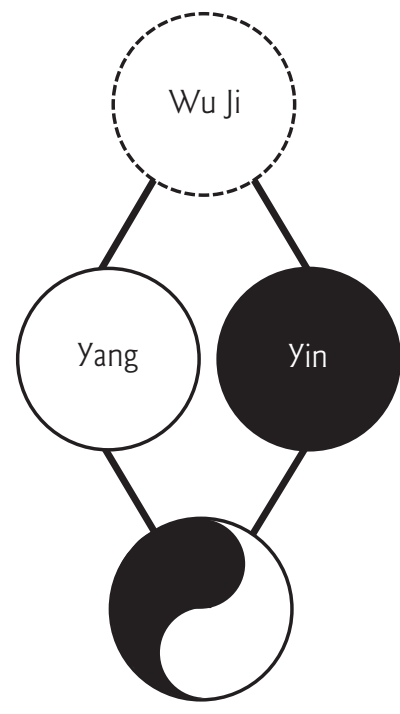

Tai Ji

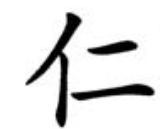

EL CARÁCTER REN ESTÁ COMPUESTO POR DOS

PARTES: A LA IZQUIERDA,

EL CARÁCTER DE LA

PERSONA; A LA DERECHA,

EL CARÁCTER DE DOS.

JUNTOS, REFLEJAN

CÓMO LAS PERSONAS SE

RELACIONAN CON

LOS DEMÁS.
Mengzi (Mencius) (371-289 a.C.) es considerado por la mayoría de historiadores como el segundo gran confuciano. Llevó el Confucianismo mas allá, añadiendo sus propias y únicas contribuciones, tales como el ideal de gobierno benigno orientado a lograr el bienestar de la gente. Mengzi comenzó considerando la mente como el centro. Pero la mente, para los confucianos, estaba del corazón humano, del cual fluye la tendencia natural de la naturaleza humana de ser bueno. Él lo explicó ejemplificando una situación: Un niño pequeño se ha apartado de su madre y va al borde de un pozo. Tú ves que está a punto de caerse. No hay nadie más ahí. ¿Qué haces? Mengzi predijo que todo el mundo trataría de salvar al niño. Existe una tendencia en la naturaleza humana hacia el bien: "La naturaleza del hombre es naturalmente buena tal como la naturaleza del agua es fluir hacia abajo" (The Mencius, 6A:2). Cómo desarrollar y dar alas a esta tendencia natural hacia la bondad es lo que enseña el Confucianismo.

El Confucianismo experimentó un renacimiento como Neo-Confucianismo durante la Dinastía Song (960-1279). Los Neo-Confucianos extrajeron su inspiración de la fuente del Confucianismo clásico. Pero también eran conocedores del Taoísmo y del Budismo. Más que oponerse a estas perspectivas, los Neo-Confucianos absorbieron conceptos que eran compatibles con los principios Confucianos. Las enseñanzas Confucianas se esparcieron por toda Asia, encontrando expresión en el gobierno, la cultura, y las artes marciales.

\section{LOS PRINCIPIOS DEL CONFUCIANISMO}

La base del pensamiento chino es una antigua creencia en una única unidad universal cósmica sin principio ni fin. El mundo fue visto como un vacío sin límites llamado Wu Ji. Del vacío se originó la actividad, el círculo vacío expresado como yang, y la inactividad, el círculo negro expresado como yin. Conjuntamente, estas interacciones de actividad e inactividad fueron llamadas Tai Ji, el famoso círculo del yin-yang. Los chinos creyeron que el universo experimenta ciclos continuos de cambio, según un extraordinario manual conocido como el Libro de los Cambios (Yi Jing). El Libro de los Cambios es considerado el primero de los Clásicos Confucianos, afianzando la teoría de ciclos universales de continua fluctuación de cambios. Fuera de este universo de cambio continuo emergieron los principios del Confucianismo: Ren, Chung Yong, Li, y Junzi.

\section{REN: El CORAZÓN HUMANo}

La naturaleza humana es dinámica, en sincronía con el universo siempre cambiante. Dadas las condiciones correctas, una semilla se convertirá en una planta sana. Del mismo modo, los seres humanos, si tiene las condiciones ambientales correctas, incluyendo el orden social debido, florecerán en adultos cooperativos, benevolentes y totalmente funcionales.

Puesto que nuestra naturaleza humana es buena, las personas poseen un conocimiento intuitivo acerca del bien y del mal. La tarea importante y algunas veces difícil es descubrirla y luego llevarla a cabo. Mengzi aconsejó: "No dejes a un hombre hacer lo que su sentido de rectitud le dice que no haga, y no dejes desear lo que su sentido de rectitud le dice que no desee. Actuar así es todo lo que tiene que hacer" (Legge, 1970: 457). Las vivencias negativas pueden inducir a las personas al mal camino, pero pueden encontrar un camino de retorno a la bondad interna mediante un cambio sincero hacia el corazón humano del ren. Viva con sinceridad de acuerdo con las mejores aptitudes de la naturaleza humana. "Ren incluye todo, no es una virtud entre otras, pero si el alma de todas las virtudes" (Jaspers, 1957: 50). 
Así, el método Confuciano para una vida feliz y satisfactoria empieza con el ren, la verdad eterna que resuena con los ritmos del corazón humano. Vivir según el ren significa vivir en sincronía y armonía con benevolencia.

La bondad humana nunca está solamente dentro del individuo, dado que estamos siempre viviendo con otros en una comunidad. La tarea es encontrar un equilibrio entre el interés propio personal y los intereses de otros. Confucio creyó que al interés propio se le presta un mejor servicio propiciando el interés común. Cuando se establece un orden correcto, todo el mundo se beneficia. La vida con ren comienza con las relaciones más cercanas. Tratando a la familia con la benevolencia del ren, se pone en movimiento una atmósfera positiva que se difunde hacia el exterior para transformar a la comunidad, al país, y finalmente al mundo entero.

\section{Chung Yong: El término medio}

A la deriva en el mar de un universo cambiante, encontramos una brújula para dirigir nuestra naturaleza humana completamente evolucionada. Este patrón humano es llamado chung yong, el término medio. El término medio es la referencia interna, el centro de la personalidad, el estándar de alineación para el auto-cultivo, positivo y activo, mostrando el Camino.

Confucio no estipuló cuál debería ser el punto central de interacción. Chung yong es el centro moderador entre los extremos para cada persona o situación. Cuanto más lejos se esté del centro, más difícil será encontrar el término medio. No cumpla cada deseo de un extremo, pero no se prive tampoco completamente. La sabiduría para cada situación se encuentra en la moderación, en el equilibrio entre los extremos.

\section{LI: El principio fundamental}

Mientras navegamos desde el chung yong, iqué acciones deberíamos realizar? Salimos a buscar el principio, $l i$, para ayudar a guiar la acción. $L i$ ha sido interpretado de muchas maneras, pero su significado siempre se refiere a la esencia, al principio, como la fuente de la forma. El principio se expresa en cosas, tales como que el principio de un avión es volar, pero también se manifiesta en la acción, en conducir relaciones sociales, y en patrones de la vida. "El principio es el trascendente [Dao] y es la raíz que produce todas las cosas" (Wittenborn, 1991: 32). Cuando el principio es comprendido por la mente, sincroniza nuestras acciones como una brújula.

La costumbre, el ritual, y la tradición fomentan y expresan el li. Por ejemplo, la devoción filial, la idea china de respeto a los padres, fortalece los lazos cariñosos y de respeto en familias y promueve la armonía social en general. La armonía social realza la armonía de la comunidad, que realza la armonía en el país entero, y finalmente expande la armonía al mundo entero. Pero li no es meramente una forma, costumbre o tradición concreta. Li es el espíritu dentro de las costumbres, los rituales o las tradiciones. El intelectual debería estudiar y participar en los rituales para entender principios básicos.

\section{Junzi: El sabio}

Junzi es un sabio, el florecimiento completo de la humanidad en su apogeo. Vivir según el li desarrolla una personalidad más fuerte. Los Junzi, fielmente al principio interno más elevado, no sólo tienen el valor de hacer lo correcto, además quieren hacerlo. "Mejor que uno que sabe qué es lo correcto es uno que tiene afición a lo correcto; y mejor que uno que tiene afición a lo correcto es uno que se deleita con lo correcto" (Giles, 1998: 60).

El sabio trata de entender el mundo explorando los principios, la misma esencia de las cosas. Entonces la comprensión obtenida nunca es superficial. El pensamiento profundo y meticuloso debe acompañar siempre al aprendizaje. El aprendizaje sin reflexión es una pérdida de tiempo. Pensar sin aprender es peligroso (parafraseado los Analects: 2-15). 
El sabio es noble en la acción. Actuar de manera benevolente, dejando a un lado preocupaciones mezquinas saca a la luz la nobleza en otros y en nosotros mismos. El bienestar y la seguridad de la benevolencia del sabio inspiran a otros a sentirse confortables y a gusto igualmente. El sabio enfatiza las cualidades positivas de otros, y les ayuda alcanzar esas cualidades. La persona inferior sólo ve las negativas.

\section{CONFUCIANISMO \& LAS ARTES MARCIALES}

Las artes marciales modernas son más que un conjunto de técnicas y habilidades. Las artes marciales son un Camino que no se limita a la destreza en la técnica como un fin en sí mismo, sino que va más allá desarrollando a la persona de un modo completo. El Camino del Confucianismo ilumina este sendero.

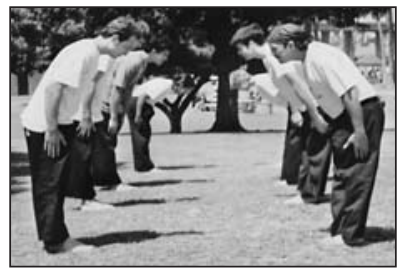

EXISTE UNA LARGA TRADICIÓN ENTRE LOS

PRACTICANTES DE ARTES MARCIALES DE COMENZAR Y TERMINAR SU PRÁCTICA CON CORTESÍA Y RESPETO.

\section{Honor y respeto}

El respeto y el honor son virtudes primarias en los artistas marciales como lo son en el Confucianismo. La clase de artes marciales comienza y acaba con cortesía, con una reverencia que muestra el respeto mutuo entre estudiantes e instructor.

Dentro de la estructura del sistema marcial existen oportunidades para expresar la virtud en la acción, el Camino Confuciano. El estudiante se inclina ante la tradición. Las artes tradicionales tienen una jerarquía de rangos de cinturón, hasta el Gran Maestro. Honrar al maestro es el equivalente a la devoción filial. El maestro es sumamente respetado. La tarea del estudiante, a lo largo de la relación de aprendizaje, es tratar de llevar a cabo respetuosa y sinceramente lo que le pide el instructor

El instructor, a su vez, honra la tradición y al estudiante con una enseñanza del estilo respetuosa, correcta, y meticulosa. El maestro enseña los patrones correctos de movimiento que los estudiantes practican para aprender los principios del estilo. La receptividad sincera y abierta de ambos, maestro y estudiante, hace posible este aprendizaje más profundo. Todos se descubren a sí mismos haciendo las cosas mejor y llevándose mejor en un compañerismo especial. Las relaciones de maestro-estudiante y de estudiante-estudiante se convierten en confianza. Todos se elevan, para volverse más sabios y mejores que como eran antes de la interacción. Los principios, aprendidos y expresados en estas relaciones ejemplares, moldean el carácter.

\section{Sinceridad}

La sinceridad es una de las virtudes más altas para el Confucianismo, como lo es para el artista marcial de hoy en día. La sinceridad propicia el aprendizaje. Los estudiantes en la escuela de artes marciales que toman su aprendizaje en serio progresan más rápidamente. Así que, si usted es un nuevo estudiante de artes marciales, sea un estudiante por completo. No sea un estudiante sólo entre semana y se olvide de su arte marcial los fines de semana. Trate de ser el mejor estudiante, con todo lo que esto debería implicar. Tome en serio las responsabilidades que son parte de aprendizaje. Cultivando el conocimiento y la sabiduría acerca de su estilo, usted llega a saber lo que se debe hacer. El esfuerzo sincero es recompensado con la mejora de las habilidades. Para los instructores, tomar su papel educativo seriamente beneficiará a los estudiantes, y en correspondencia usted se beneficiará también. Usted recibe las satisfacciones que provienen de estar inmerso en el progreso de cada estudiante. La vida continúa desarrollándose en sus ciclos permanentemente cambiantes, tal como el Libro de los Cambios predice, mientras usted y los otros miembros de su clase maduran para vivir en plenitud. 


\section{Encontrar el Centro para mejorar la técnica}

La práctica de muchas técnicas básicas de mano y de pie vienen de y pasan por el centro con equilibrio. Los puñetazos se realizan hacia el centro, los bloqueos se dirigen al centro o desde el centro. Las patadas también se dirigen hacia el centro.

Así como Confucio no dijo a la gente lo que debería ser el centro para todas las personas, los artistas marciales deben descubrir su propio centro. La localización del centro depende de la constitución. Una persona alta y corpulenta tendrá una longitud y altura diferente para centrar su postura que un individuo pequeño y delgado.

Hay muchas técnicas diferentes que aprender, cada una con varias posibilidades de aplicación. Por ejemplo, una postura retrasada tiene un punto de equilibrio diferente que una postura avanzada. Un puñetazo a un blanco elevado se centra de diferente forma que un puñetazo a un blanco bajo. Aunque todas las técnicas comparten un principio central común: chung. Descúbralo y permanezca cerca de él. Por ejemplo, los principiantes cometen el error de sacar el puño desde el hombro, pero hacia un lado, fuera de su línea central. Como consecuencia, la fuerza potencial que puede generarse desde el cuerpo entero se pierde. El puñetazo viene simplemente del brazo y hombro, resultando una técnica mucho más débil. Su meta personal debe incluir todo lo que usted es, su cuerpo entero, trabajando como una unidad equilibrada, para lograr la máxima potencia, agilidad, y velocidad.

\section{La práctica de rutinas: $\mathrm{Li}$ en movimiento}

Las rutinas son patrones de movimientos transmitidos de generación en generación, la literatura clásica del estilo. Como la realización de un ritual confuciano, la práctica de rutinas transmite el espíritu tradicional del arte y ofrece al estudiante un patrón para la perfección. Las nuevas comprensiones y las habilidades perfeccionadas se desarrollan cuando usted honra la rutina a través de una práctica precisa y meticulosa.

Para entrar en la práctica de la rutina como un ritual, empiece la práctica con la colocación exacta de manos y pies. Tómese un momento para estar tranquilo, dirigiendo todo pensamiento a la rutina y sólo a la rutina. Espere hasta que su mente esté tranquila y lista. Luego realice los movimientos con la atención centrada a cada movimiento. Para finalizar, regrese exactamente a la posición final correcta.

Cuando los estudiantes aprenden a realizar correctamente una rutina, comienzan a tener una comprensión más profunda de los patrones y significados a varios niveles que entrañan las rutinas. El aprendizaje proviene del li codificado en los movimientos. Así, la comprensión obtenida es necesariamente no superficial si el aprendizaje de las rutinas es acompañado por un pensamiento profundo y meticuloso. Cuando usted entiende el principio, las aplicaciones llegarán. Por ejemplo, piense en un bloqueo sencillo de una de sus rutinas. ¿Cómo es que es insustituible? ¿Cómo es que expresa el principio del bloqueo en general? Después, considere los movimientos que componen los movimientos, tal como sucede en el bloqueo del filo de un cuchillo, y usted descubrirá otros principios de movimiento, lo suave dentro de lo duro, o lo duro escondido en lo suave. Usted puede observar cualquier movimiento particular como un ejemplo del li, una fuente para diversas aplicaciones y modificaciones creativas.

\section{Oportunidades para el Ren}

La benevolencia es la mente y la justicia del camino. Los artistas marciales desarrollan un sentido de justicia. Aunque desarrollan la fuerza con el entrenamiento, aprenden a usarla sabiamente. A través de la práctica estable, disciplinada, el entrenamiento imbuye a los practicantes con determinación. A través del acto mismo, el valor ético cobra vida. Las artes marciales tradicionales de hoy día incorporan este sentido de justicia en los códigos

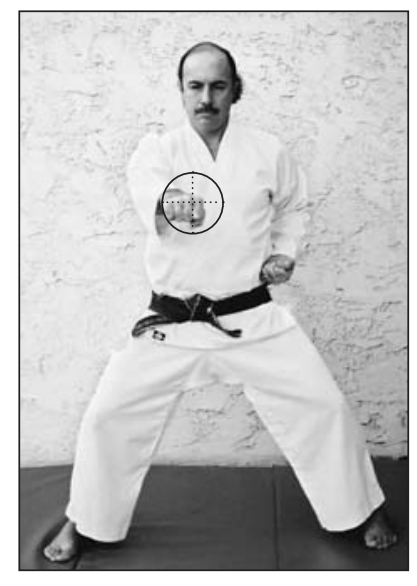

El PUÑo PARTE DEL CENTRO PARA MAXIMIZAR LA FUERZA.

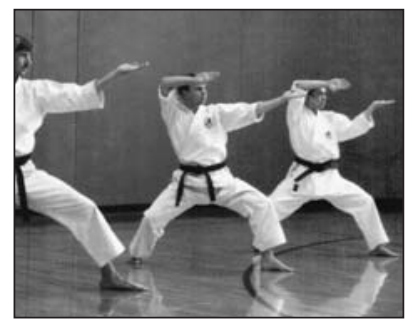

LA PRÁCTICA DE RUTINAS ES EL LI EN MOVIMIENTO

Fotografía cortesía de

Wayne Van Horne.

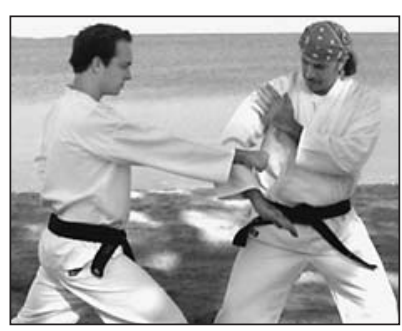

Contempla el Gesto de UNO DE LOS MOVIMIENTOS EN LA PRÁCTICA DE UNA RUTINA, COMO ES UN BLOQUEO DE CUCHILLO. Oculto en el BLOQueo, EL MOVIMIENTO DENTRO DEL MOVIMIENTO ES UN SUAVE BLOQUEO. 


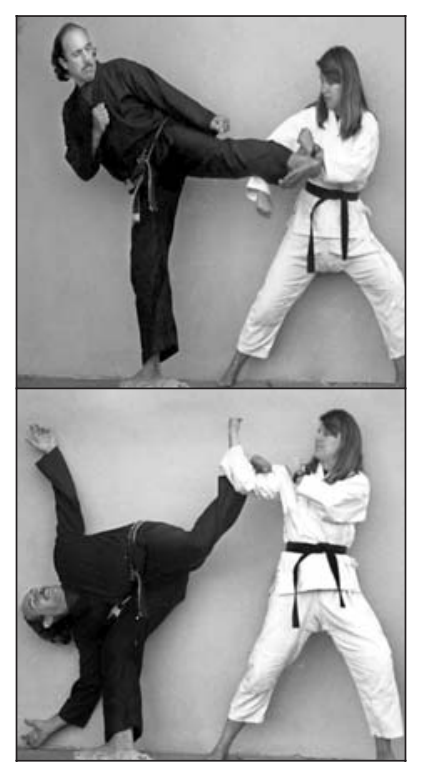

CON LA CONTEMPLACIÓN

DEL LI, DESCUBRIRÁ MUCHAS

VARIACIONES OCULTAS.

AQUÍ SE EJEMPLIFICA UNA POSIBILIDAD DE DERRIBO CON UN MOVIMIENTO DE BLOQUEO.

éticos que adoptan los estudiantes: usar su arte marcial sólo para la defensa, no ser nunca los que inician un ataque. Implícita en este código está la benevolencia que subyace al ren. Las rutinas comienzan con un bloqueo, no con un ataque, como una manifestación concreta de este principio.

Los artistas marciales espiritualmente desarrollados hacen todo lo que pueden para evitar peleas banales. Desarrollando un carácter fuerte, ellos no se involucran fácilmente en conflictos. El principio de la defensa es impedir dañarse ellos mismos y a los demás, resguardando por consiguiente la armonía. Estos fuertes sentidos de benevolencia y justicia son los principios que guían al artista marcial en la acción.

Cuando buscamos verdaderamente lo positivo en nuestras amistades y colegas, permitimos y además promovemos sus mejores cualidades. El artista marcial experto es capaz de prolongar el ren incluso bajo un ataque. Esta historia verdadera lo ilustra: Un joven con un nunchaku andaba buscando pelea. Comenzó a provocar y amenazar a un desconocido inocente, pero siendo desconocido para el joven era de hecho un practicante de taekwondo de alto rango. En vez de dejarse provocar, el artista marcial serenamente invitó al asaltante a usar su mente en lugar de su nunchaku. Sin revelar su habilidad física, el artista marcial preguntó tranquilamente: "¿Para que pelear? Creo que las personas luchan por debilidad. La persona verdaderamente fuerte puede hablar a fondo las cosas y resolver las diferencias. Quizá haya un malentendido. Arreglémoslo en lugar de luchar".

Al principio, el asaltante se enojó aún más, pero como el artista marcial habló de forma sincera, serena y segura, el asaltante se detuvo, oyó, y lo reconsideró. Como Confucio habría predicho, el asaltante guardó al fin su arma y habló del problema. Cuando producimos como respuesta inclinaciones positivas en los otros, permitimos que éstas emerjan y además promovemos sus mejores cualidades.

\section{Perfeccionarse durante toda la vida a través del aprendizaje}

Las artes marciales deberían ser estudiadas con la mente puesta en la meta de la perfección: la perfección de la forma en movimiento, la perfección de la mente en la actividad, la perfección del principio dentro del sistema. Por consiguiente, para lograr el dominio verdadero, estudie el arte hasta sus raíces. Fortalezca y extienda las raíces, y muchas ramas le seguirán con naturalidad. Con el dominio del estilo, el artista marcial se convierte en un junzi con una comprensión profunda que se generaliza y extiende hacia la vida.

El sabio reconoce que siempre hay más que aprender. El aprendizaje en las artes marciales es un proceso paso a paso, avanzando a través de un orden correcto. Pero el aprendizaje es infinito, incesante a lo largo de toda la vida. El cinturón negro se convierte en una parte identitaria del artista marcial, en una actitud para acercarse a los desafíos de la vida con fuerza, ánimo, disciplina y respeto. Los practicantes competentes saben que obtener un cinturón negro no es el fin, sólo es el comienzo. El camino para la fuente de virtud reside dentro de cada persona. Entrénese duro y encontrará el Camino de la sabiduría.

\section{Conclusión}

El Confucianismo nos anima a que seamos seres humanos completamente desarrollados, no sólo para el desarrollo personal sino también para el desarrollo de los demás. Hay esperanza, no importa cuán duro pueda parecer, de que si mejoramos, el mundo mejorará. Confucio dijo que el hombre de virtud moral, teniendo el deseo de aguantar con firmeza, da la firmeza a los otros. A través de una práctica estable y sincera de las artes marciales puede encontrarse el principio, induciendo a la maestría, no solo en las técnicas, sino también en la vida. 


\section{BIBLIOGRAFÍA}

Bell, D. \& Chaibong, H. (2003). Confucianism for the modern world. Cambridge, England: Cambridge University Press.

De BARY, W. (2004). Nobility and civility: Asian ideals of leadership and the common good. Cambridge, MA: Harvard University Press.

De Bary, W., \& Bloom, I. Eds. (1999). Sources of Chinese tradition from earliest times to 1600. New York: Columbia University Press.

De Bary, W., \& Lufrano, R. (EDs.). (2000). Sources of Chinese tradition from 1600 to the twentieth century. New York: Columbia University Press.

Chan, W. (1989). Chu Hsi - New studies. Honolulu: University of Hawaii Press.

CHAn, W. (1963). A sourcebook of Chinese philosophy. Princeton: Princeton University Press.

CHAN, W. (1963b). Instructions for practical living and other Neo-Confucian writings by Wang Yang-ming. New York: Columbia University Press.

Glosario

Dao 道

junzi 君子

Kong Fuzi 孔夫子

Kong Qiu 孔丘

Kong Zi 孔子

li 理

Mengzi 孟子

ren 仁

tai ji 太極

wu ji 無極

yi Jing 易經

zhong yong 中庸

Chang, Chi-yun (1957). The life of Confucius. Taipei, Taiwan: China News Press.

Dobson, W. (1969). Mencius. London: Oxford University Press.

Giles, L. (1998). The sayings of Confucius. Middlesex, England: Tiger Books.

Jaspers, K. (1962). Socrates, Buddha, Confucius, Jesus. San Diego: Harvest/HBJ Book.

Legge, J. (1971). Confucius. New York: Dover Publications.

Legge, J. (1970). The works of Mencius. New York: Dover Publications.

Miller, F. \& Keyt, D. (Eds.). (1991). A companion to Aristotle's Politics. Oxford: Blackwell.

Pound, E. (1951). Confucius: The great digest and unwobbling pivot. New York: New Directions Books.

Simpkins, C. A. \& Simpkins, A. (2005). Taekwondo: Building on the basics. San Diego, CA: Radiant Dolphin Press.

Simpkins, C. A. \& Simpkins, A. (2004). Meditation from thought to action with audio CD. Diego, CA: Radiant Dolphin Press.

Simpkins, C. A. \& Simpkins, A. (2004). Buddhism in ten. Boston: Tuttle Publishing.

Simpkins, C. A. \& Simpkins, A. (2003). Chung Do Kwan: The power of Taekwondo. Boston: Tuttle Publishing.

Simpkins, C. A. \& Simpkins, A. (2001). Simple Buddhism: A guide to enlightened living. Boston: Tuttle Publishing.

Simpkins, C. A. \& Simpkins, A. (2000). Simple Confucianism: A guide to living virtuously. Boston: Tuttle Publishing.

Simpkins, C. A. \& Simpkins, A. (1999). Simple Taoism: A guide to living in balance. Boston: Tuttle Publishing.

Tsunetomo, Y. (1979). Hagakure: The book of the samurai. Tokyo: Kodansha.

Watson, B. (1969). Hsun Tzu. New York: Columbia University Press.

Wilhelm, R. (1931). Confucius and Confucianism. New York: Harcourt, Brace, and Javonovich.

Wittenborn, A. (1991). Further reflections on things at hand: A reader Chu Hsi. Lantham, Maryland: University Press America.

Wong, R. (1997). China transformed: Historical change and the limits of European experience. Ithaca, New York: Cornell University Press.

Yao, X. 2000. An introduction to Confucianism. Cambridge, England: Cambridge 